\title{
Super-twisting sliding mode based nonlinear control for planar dual arm robots
}

\author{
Tung Lam Nguyen, Huu Tinh Vu \\ School of Electrical Engineering, Hanoi University of Science and Technology, Vietnam
}

\begin{tabular}{|c|c|}
\hline Article Info & ABSTRACT \\
\hline Article history: & In this paper, a super-twisting algorithm sliding mode controller is proposed for a \\
\hline Received Jan 22, 2020 & planar dual arm robot. The control strategy for the manipulator system can effec- \\
\hline Revised Mar 10, 2020 & tively counteract chattering phenomenon happened with conventional sliding mode \\
\hline Accepted Apr 12, 2020 & $\begin{array}{l}\text { approach. The modeling is implemented in order to provide the capability of mane } \\
\text { vering object in translational and rotational motions. The control is developed for }\end{array}$ \\
\hline Keywords & $\begin{array}{l}\text { 2n-link robot and subsequently simulations is carried out for a } 4 \text {-link system. Com- } \\
\text { parative numerical study shows that the designed controller performance with good }\end{array}$ \\
\hline Dual arm robot & racking ability and smaller chattering compared with basic sliding mode controller. \\
\hline
\end{tabular}

Nonlinear control

Siding mode control

Super-twisting

This is an open access article under the $\underline{C C B Y-S A}$ license.

\section{Corresponding Author:}

Tung Lam Nguyen,

School of Electrical Engineering,

Hanoi University of Science and Technology, Ha Noi, Vietnam.

Email: lam.nguyentung@hust.edu.vn

\section{INTRODUCTION}

Initially, robots are designed with an idea of developing a mechanism to replace/support human when interacting with the environment [1-4]. Along with the speedy evolution of control engineering, computer science, and mechanical technologies, robots have an inclination toward possessing the human form [5-7]. One of the earliest humanoid robots but currently attracted researcher's attentions is a dual arm manipulator [8]. In comparison to a single arm manipulator, the dual arm manipulator is capable of handling sizable objects, objects that having a certain degree of flexibility, and assembling mechanical parts [9]. In recent years, with the development of modern dual-arm robots (DAR), this concept becomes more and more important for the automation of processes in the life sciences [10]. Due to the human-like structure of the robotic arms, it can used for a wide number of application.

Various applications of in automatic measurement field in [11-13]. The other applications are also used in industrial manipulation tasks for performing automatic assembly operations [14, 15], or using for automation of cell production system for cellular phones [16]. Primarily designed to work in coordinated operations, the dual arm manipulator suffers from inherent kinematic and strong dynamic couplings. The phenomenon poses a challenge in dual arm manipulator control problems. The situation become even worse when the bi-manual manipulator holding a rigid object due to the formation of closed kinematic chains. Individual arms are dynamically engaged through the manipulated object, thus in coordinated modes, two arms operate in constrained motions. This leads to a requirement of complex control schemes that can handle the manipulator and object dynamics. Various control methods have been developed for the . Classical algorithms as X. Yun at el. [17] apply nonlinear feedback techniques approach, N. Sarkar at el. [18] propose control scheme integrates hybrid position/force control and vibration suppression. In paper [19], a fuzzy force control framework is 
proposed for dual-industrial robot systems. Besides, there are also many researches consider adaptive control algorithms for the . Zhi Liu at el. [20] use an adaptive neural control scheme to deal with the problem of output hysteresis. N. Yagiz at el. [21] develop a sliding mode controller to investigate the performance of two coordinated planar arms in transporting a load to its new location with friction-assisted handling. In [22], the authors propose a sliding mode control combined with a parameter estimator for the robot system. The result show a practical approach since many parameters are not convenient to measure. In this study, we develop a generalized model for model with $2 n$ degrees of freedom and introducing a control approach for trajectory tracking using super-twisting algorithm. The feasibility of the control is confirmed using the 4 DoF model to numerically investigate tracking quality and the adaptability to the disturbance of the super-twisting sliding mode controller.

\section{MATHEMATICAL MODEL}

The configuration of model consisting of two planar arm robot that is shown in Figure 1. Each arm robot is composed of a set of links connected together by revolute joints. In this study, the model with $2 n$ joints is assumed through that all joints have only a single Degree-of-Freedom (DoF). With the $i^{\text {th }}$ joint, we associate a joint variable, denoted by $\theta_{i}$. Additional, $m_{i}, I_{i}, L_{i}$ represente the mass, mass moment of inertia and length of related links, respectively. The mass of load is denoted by $m(t)$, and $, d_{1}, d_{2}$ denote the width of the rectangular load and the distance between the bases of the robot arms. There is viscous frictions acting on all of the joints denoted by $b_{i}$.

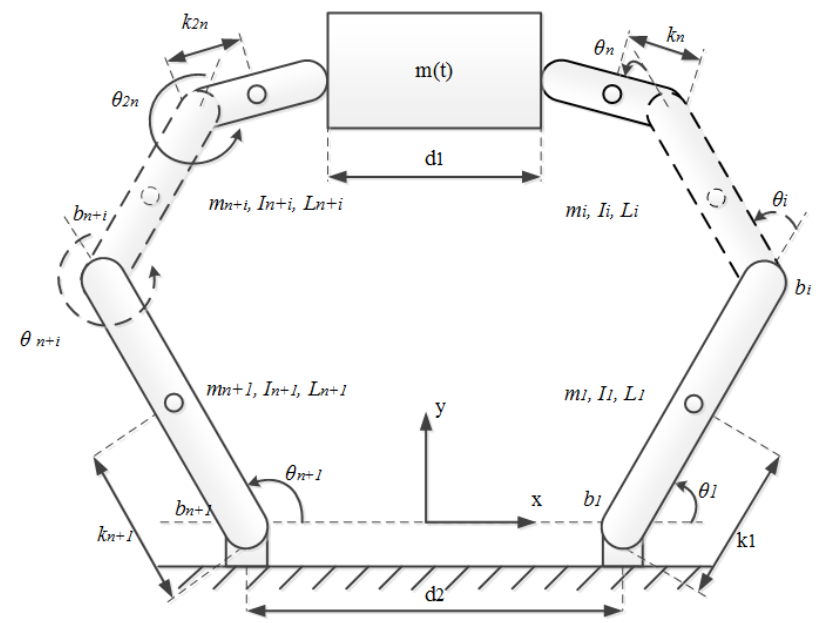

Figure 1. model coordinates

The forward kinematic problem is to determine the position and orientation of the end-effector, given the values for the joint variable of the robot. To perform the kinematic analysis of $2 n$ links model, we represent used Denavit-Hartenberg conventions that can be referred to [23]. By using the generalized frame oxyz which is shown in Figure 1, the kinematic equations of right arm robot are

$$
\left\{\begin{array}{l}
x_{n}=L_{1} \cos \left(\theta_{1}\right)+L_{2} \cos \left(\theta_{1}+\theta_{2}\right)+\ldots+L_{n} \cos \left(\theta_{1}+\theta_{2}+\ldots+\theta_{n}\right)+\frac{d_{2}}{2} \\
y_{n}=L_{1} \sin \left(\theta_{1}\right)+L_{2} \sin \left(\theta_{1}+\theta_{2}\right)+\ldots+L_{n-1} \sin \left(\theta_{1}+\theta_{2}+\ldots+\theta_{n}\right)
\end{array}\right.
$$

Kinematic equations of left arm robot in the generalized frame oxyz are

$$
\left\{\begin{array}{l}
x_{2 n}=L_{n+1} \cos \left(\theta_{n+1}\right)+L_{n+2} \cos \left(\theta_{n+1}+\theta_{n+2}\right)+\ldots+L_{2 n} \cos \left(\theta_{n+1}+\theta_{n+2}+\ldots+\theta_{2 n}\right)-\frac{d_{2}}{2} \\
y_{2 n}=L_{n+1} \sin \left(\theta_{n+1}\right)+L_{n+2} \sin \left(\theta_{n+1}+\theta_{n+2}\right)+\ldots+L_{2 n} \sin \left(\theta_{n+1}+\theta_{n+2}+\ldots+\theta_{2 n}\right)
\end{array}\right.
$$




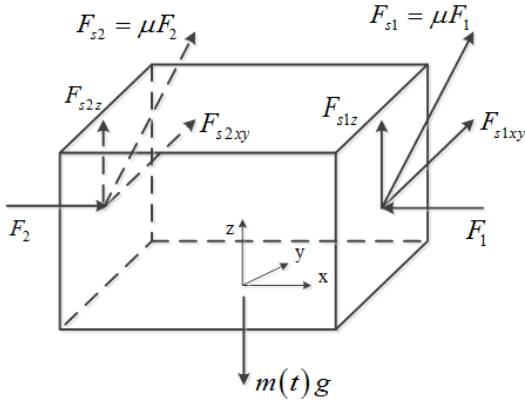

(a) When the load at fixed position

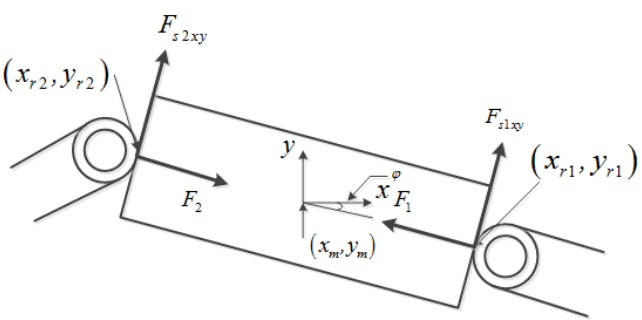

(b) When the load at fixed position

Figure 2. Representation of forces, (a) When the load at fixed position and (b) When the load at fixed position

Where as the kinematic equations describe the motion of the robot, the dynamic equations explicitly describe the relationship between force and motion. Firstly, we concerned about the term of dynamic which handling the load. Representation of forces acting on load is illustrated in Figure 2 (a). Where $F_{1}, F_{2}$ are forces of right arm, left arm tips to the load. The friction forces $F_{s 1} F_{s 2}$ and their components $F_{s 1 x y}, F_{s 2 x y}, F_{s 1 z}$, $F_{s 2 z}$ between the arm tips and the load surface. The force $F_{1}, F_{2}$ can be write in frame oxy as

$$
\begin{aligned}
& F_{1 x}=-F_{1} \cos \varphi-F_{s 1 x y} \sin \varphi, \quad F_{1 y}=-F_{1} \sin \varphi+F_{s 1 x y} \cos \varphi \\
& F_{2 x}=F_{2} \cos \varphi-F_{s 2 x y} \sin \varphi, \quad F_{2 y}=F_{2} \sin \varphi+F_{s 2 x y} \cos \varphi
\end{aligned}
$$

Next, we used the Euler-Lagrange equations to derive the dynamical equations in the $n$-link robot. Following [23], the motion equation of model in matrix form as

$$
D(\mathbf{q}) \ddot{\mathbf{q}}+C(\mathbf{q}, \dot{\mathbf{q}}) \dot{\mathbf{q}}-\mathbf{g}(\mathbf{q})=\tau
$$

when the robot perform the transportation, the motion of robot can be effect by external disturbances, viscous friction forces and load torques. The motion equation of model after handling the load were given as

$$
D(\mathbf{q}) \ddot{\mathbf{q}}+C(\mathbf{q}, \dot{\mathbf{q}}) \dot{\mathbf{q}}-\mathbf{g}(\mathbf{q})=\tau+\tau_{0}-\omega-\beta
$$

where state variables $\mathbf{q}=\left[\theta_{1}, \theta_{2}, \ldots, \theta_{2 n}\right]^{T} ; \tau_{0}$ is the load torque $; \omega, \beta$ is $2 n \times 1$ vectors represent for the external disturbance torques and the viscous friction forces, respectively. We specified as

$$
\omega=\left[\omega_{1}, \omega_{2}, \ldots, \omega_{2 n}\right]^{T}, \quad \beta=\left[b_{1} \dot{\theta}_{1}, b_{2} \dot{\theta}_{2}, \ldots, b_{2 n} \dot{\theta}_{2 n}\right]^{T}
$$

\section{CONTROLLER DESIGN}

\subsection{Sliding mode controller}

The state space form of model with joint variables $\mathbf{q}=\left[\theta_{1}, \theta_{2}, \ldots, \theta_{2 n}\right]^{T}$ can be written as

$$
\ddot{\mathbf{q}}=D(\mathbf{q})^{-1}\left[\tau_{0}-\omega-\beta-C(\mathbf{q}, \dot{\mathbf{q}})-\mathbf{g}(\mathbf{q})\right]+D(\mathbf{q})^{-1} \tau
$$

The state space form of model can be rewritten as form of a non-linear dynamic system

$$
\dot{x}=f(x, t)+B(x, t) u
$$

Where the state variables in the state space $x \in \mathbb{R}^{4 n}, x=[\mathbf{q}, \dot{\mathbf{q}}]^{T}=\left[\theta_{1}, \theta_{2}, \ldots, \theta_{2 n}, \dot{\theta}_{1}, \dot{\theta}_{2} \ldots, \dot{\theta}_{2 n}\right]^{T}$. The state equations without the control inputs $f(x, t) \in \mathbb{R}^{4 n}, f(x, t)=\left[\dot{\theta}_{1}, \dot{\theta}_{2} \ldots, \dot{\theta}_{2 n}, f_{1}, f_{2}, \ldots, f_{2 n}\right]^{T}$ with the component $\left[f_{1}, f_{2}, \ldots, f_{2 n}\right]^{T}=D(\mathbf{q})^{-1}\left[\tau_{0}-\omega-\beta-C(\mathbf{q}, \dot{\mathbf{q}})-\mathbf{g}(\mathbf{q})\right]$. Meanwhile, $u \in \mathbb{R}^{2 n}$ is generalized torque input vector and $B=[\operatorname{zeros}(2 n), D(\mathbf{q})]^{T} \in \mathbb{R}^{4 n \times 2 n}$ is the elements of which are the coefficients of the 
generalized control inputs in the state equations. With $\Delta(x)=x_{r}-x$ are the difference between the reference value and the system response. We define the sliding surface in the state space form as

$$
s=[G] \Delta(x)=[G]\left[\begin{array}{ll}
e & \dot{e}
\end{array}\right]^{T}
$$

Where $s \in \mathbb{R}^{4 n}, \lambda_{i}(i=1,2, \ldots, 2 n)$ are the coefficients of sliding surface, and $[G] \in \mathbb{R}^{2 n \times 4 n}$ as

$$
[G]=\left[\begin{array}{cccccccc}
\lambda_{1} & 0 & 0 & 0 & 1 & 0 & 0 & 0 \\
0 & \lambda_{2} & 0 & 0 & 0 & 1 & 0 & 0 \\
0 & 0 & \ddots & 0 & 0 & 0 & \ddots & 0 \\
0 & 0 & 0 & \lambda_{2 n} & 0 & 0 & 0 & 1
\end{array}\right]
$$

In sliding mode controlled systems, the control input is changed to drives, and maintains the system states on a sliding surface[24], [25]. Therefore, consider the control law $\mathrm{u}=\mathrm{u}_{e q} \quad(s=0)$ $u_{N} \quad(s \neq 0)$

Where the equivalent control $u_{e q}$ is the value the switched signal to maintain sliding, and which is formally obtained as the solution to the algebraic equation $\dot{s}=0$ when $s=0$, must satisfy

$$
\begin{gathered}
\dot{s}=[G] \dot{x}_{r}-[G]\left[f(x, t)+B(x, t) u_{e q}\right]=0 \\
u_{e q}=[G B(x, t)]^{-1}\left[G \dot{x}_{r}-G f(x, t)\right]
\end{gathered}
$$

Let define Lyapunov candidate function has to be positive definite $V(s)=\frac{1}{2} s^{T} s$. To drive $x$ reach the sliding surface the $u_{N}$ signal have to make sure $\dot{V}(s)=s^{T} \dot{s}<0$ for $s \neq 0$. Therefore, $u_{N}=u_{e q}+\Delta$ we have

$$
\begin{aligned}
\dot{s} & =[G] \dot{x}_{r}-[G]\left[f(x, t)+B(x, t)\left(u_{e q}+\Delta\right)\right] \\
& =-[G] B(x, t) \Delta=0
\end{aligned}
$$

If we denoted that $\dot{s}=-[K] \operatorname{sign}(s)$ with

$$
\begin{gathered}
{[K]=\left[\begin{array}{cccc}
k_{1} & 0 & 0 & 0 \\
0 & k_{2} & 0 & 0 \\
0 & 0 & \ddots & 0 \\
0 & 0 & 0 & k_{2 n}
\end{array}\right]} \\
\Delta=[G B(x, t)]^{-1}[K] \operatorname{sign}(s)
\end{gathered}
$$

Combining with (12), the total control input is found by

$$
u=[G B(x, t)]^{-1}\left[G \dot{x}_{r}-G f(x, t)+[K] \operatorname{sign}(s)\right]
$$

\subsection{Super-twisting sliding mode controller}

The sliding set of order $r$ defined by $s=\dot{s}=\ddot{s}=\ldots=s^{(r-1)}=0$. The first-order sliding mode tries to keep $s=0$, second-order sliding mode verified $s=\dot{s}=0$. We purpose $\left[z_{1}, z_{2}\right]^{T}=[s, \dot{s}]^{T}$, the problem of second-order sliding mode is reduced to the stabilization in finite time of the auxiliary system as below

$$
\left\{\begin{array}{l}
\dot{z_{1}}=z_{2} \\
\dot{z_{2}}=\varphi(x, t, u)+\gamma(x, t) \dot{u}
\end{array}\right.
$$


Where,

$$
\left\{\begin{array}{l}
\varphi(x, t, u)=\frac{\partial \dot{s}}{\partial t}+\frac{\partial \dot{s}}{\partial x}[f(x, t)+B(x, t) u] \\
\gamma(x, t)=\frac{\partial \dot{s}}{\partial x} B(x, t)
\end{array}\right.
$$

The super-twisting algorithm to stabilize the robot manipulator requested that for some positive constants $q, C, K_{1}, K_{2}, U$, and,

$$
|\dot{\varphi}(\cdot)|+U|\dot{\gamma}(\cdot)| \leq C, \quad 0<K_{1} \leq \gamma(\cdot) \leq K_{2}, \quad\left|\frac{\varphi(\cdot)}{\gamma(\cdot)}\right|<q U, 0<q<1
$$

The super-twisting sliding mode controller is given by

$$
u_{s w}=-\beta \sqrt{\left|z_{1}\right|} \operatorname{sign}\left(z_{1}\right)+u_{1}
$$

$1=-u \quad(|u|>U)$

$\alpha \operatorname{sign}\left(z_{1}\right) \quad(|u| \leq U)$ The effective control signal $u$ for super-twisting algorithm consists of two terms: $u=$ $u_{e q}+u_{s w}$. Similar sliding mode controller, $u_{e q}$ is equivalent control signal that guarantees $s=\dot{s}=0$ or $\dot{s}+\lambda s=0$

$$
\begin{aligned}
& {[G] \dot{x}_{r}-[G]\left[f(x, t)+B(x, t) u_{e q}\right]+\lambda s=0} \\
& u_{e q}=[G B(x, t)]^{-1}\left(\left[G \dot{x}_{r}-G f(x, t)\right]+\lambda s\right)
\end{aligned}
$$

The term of $u_{s w}$ is control signal of super-twisting algorithm. Hence, combining (20) and (22) the supertwisting algorithm have the control input

$$
u=[G B(x, t)]^{-1}\left(\left[G \dot{x}_{r}-G f(x, t)\right]+\lambda s\right)-\beta \sqrt{\left|z_{1}\right|} \operatorname{sign}\left(z_{1}\right)+u_{1}
$$

\section{RESULT AND DISCUSSION}

\subsection{Trajectory planning}

To illustrate the effectiveness and applicability of super-twisting sliding mode controller, we design the super-twisting sliding mode controller for model with four DoF $(n=2)$, and simulation results are compared with conventional sliding mode We used point-to-point motion for the trajectory planning, which is specified initial and final configuration of end-effector. In this case, the inverse kinematic solution must be used to convert end-effector configuration to a sequence of joint configurations. The reference trajectories are given by

$$
\begin{array}{ll}
\left.x_{1}(t)=x_{f 1}+\left(x_{i 1}-x_{(} f 1\right)\right) e^{-10 t^{2}} & \left.y_{1}(t)=y_{f 1}+\left(y_{i 1}-y_{(} f 1\right)\right) e^{-10 t^{2}} \\
\left.x_{2}(t)=x_{f 2}+\left(x_{i 2}-x_{(} f\right)\right) e^{-10 t^{2}} & \left.y_{2}(t)=y_{f 2}+\left(y_{i 2}-y_{(} f 2\right)\right) e^{-10 t^{2}}
\end{array}
$$

Where $x_{1}(t), y_{1}(t), x_{2}(t), y_{2}(t)$, are the reference trajectories of right arm and left arm robot; $x_{i 1}, y_{i 1}, x_{i 2}, y_{i 2}$, are the start positions, $x_{f 1}, y_{f 1}, x_{f 2}, y_{f 2}$, are the destination positions. Each periods, the robot represents the amount of time $t=2 \mathrm{~s}$. In last period reach to the final configuration, the robot must to rotate $\varphi=-(\pi / 4)+(\pi / 4) e^{-10(t-4)^{2}}$. Hence, the reference trajectories in this period $t=4 \rightarrow 6 \mathrm{~s}$ is

$$
\begin{array}{ll}
x_{1}(t)=x_{m}+\left(x_{i 1}-x_{m}\right) e^{-10 t^{2}}+\frac{d_{1}}{2} \cos \varphi & y_{1}(t)=y_{m}+\left(y_{i 1}-y_{m}\right) e^{-10 t^{2}}+\frac{d_{1}}{2} \sin \varphi \\
x_{2}(t)=x_{m}+\left(x_{i 2}-x_{m}\right) e^{-10 t^{2}}-\frac{d_{1}}{2} \cos \varphi & y_{2}(t)=y_{m}+\left(y_{i 2}-y_{m}\right) e^{-10 t^{2}}-\frac{d_{1}}{2} \sin \varphi
\end{array}
$$




\subsection{Numerical simulation}

From Figure 3(a), we can see that, both the basic sliding mode controller and super-twisting sliding mode controller solve successfully the problem of trajectory tracking. The actual value of the rotation angle at the joints are very closely track to the reference value. Besides, we also find that the quality of super-twisting controller is better when the maximum deviation angle at joints is only about $0.4 \times 10^{-3}$ rad, while in sliding mode controller is about $0.9 \times 10^{-3} \mathrm{rad}$. The value of rotation of the object respect to the $o_{x}$ axis in both controllers is very close to the set value. In the period from 0 to 4 seconds this deviation angle is equal to 0 degrees, and starts from the 4th second onward. The object begins to rotate with the trajectory as described in above, until the end of the cycle at the 6th second the object's rotation angle respect to $o_{x}$ is 45 degrees. The deviation made by the basic sliding controller is clearly larger the deviation made by the super-twisting sliding mode controller.
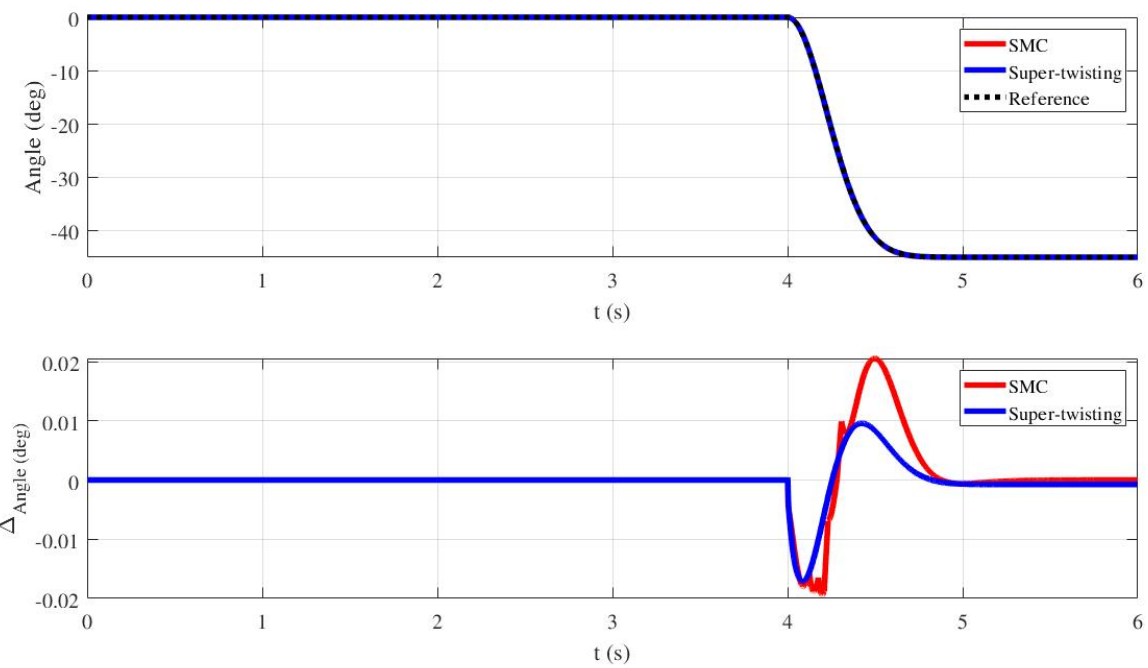

(a) The reference and the actual joint angles
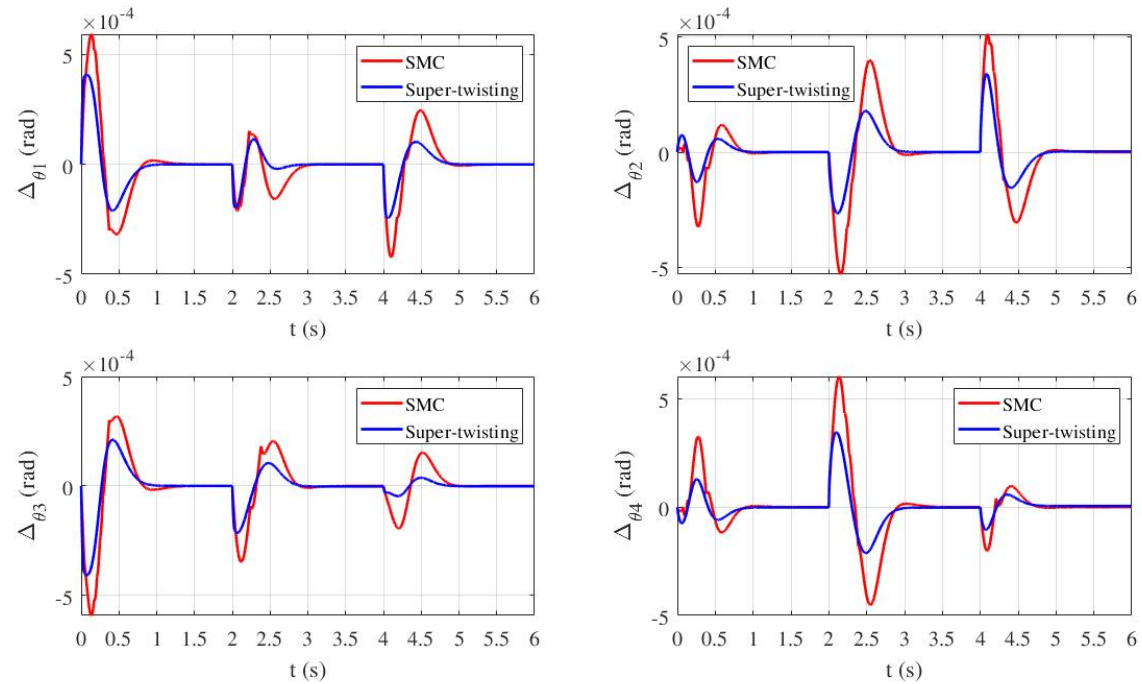

(b) Error tracking of the joint variables

Figure 3. The reference and the actual joint angles, (a) The reference and the actual joint angles,

(b) Error tracking of the joint variables

The Figures 4 (a) and (b) depict the torque of joints and the interaction forces between the robot arm and the object, respectively. The control torque signals as well as the interaction forces from the basic sliding mode controller are greatly vibrated (chattering), while in the super-twisting sliding mode controller, 
the vibration range and vibration amplitude are very small. Also from the simulation results we see that the force acting on the object is match with the requirement to move the object according to the trajectory. From second to 4th second, force $F_{2}$ is greater than $F_{1}$ to move objects from left to right; the forces $F_{s 1 x y}$ and $F_{s 2 x y}$ are equally assured so that the object does not rotate in the direction of $o_{z}$ because at this time the object is moving in parallel with the $o_{x}$ axis. From the 4th to the 6th seconds, during this period the object is rotating so that the forces $F_{s 1 x y}$ and $F_{s 2 x y}$ are no longer equal. Summary, in the absence of noise, the quality of the super-twisting sliding mode controller is better than the basic sliding mode controller. We assume the noise signal is a function of time $\omega=\left[\begin{array}{lll}\sin 10 \pi t & \sin 20 \pi t-\sin 10 \pi t-\sin 20 \pi t\end{array}\right]^{T}$. It can be seen that both controllers are affected by noise, especially in the basic sliding mode controller, when the noise appears, the vibration amplitude and frequency of the control torque signal and the interaction force also increase. For supertwisting controller, the effect of noise is much smaller than the basic sliding mode controller. In general, both two controllers response well to the the requirements of trajectory control. However, in terms of chattering reduction and feasibility in practical, the super-twisting sliding mode controller will be more dominant than the basic sliding mode controller as shown in Figure 5 and The numerical simulation values can be found in Table 1.
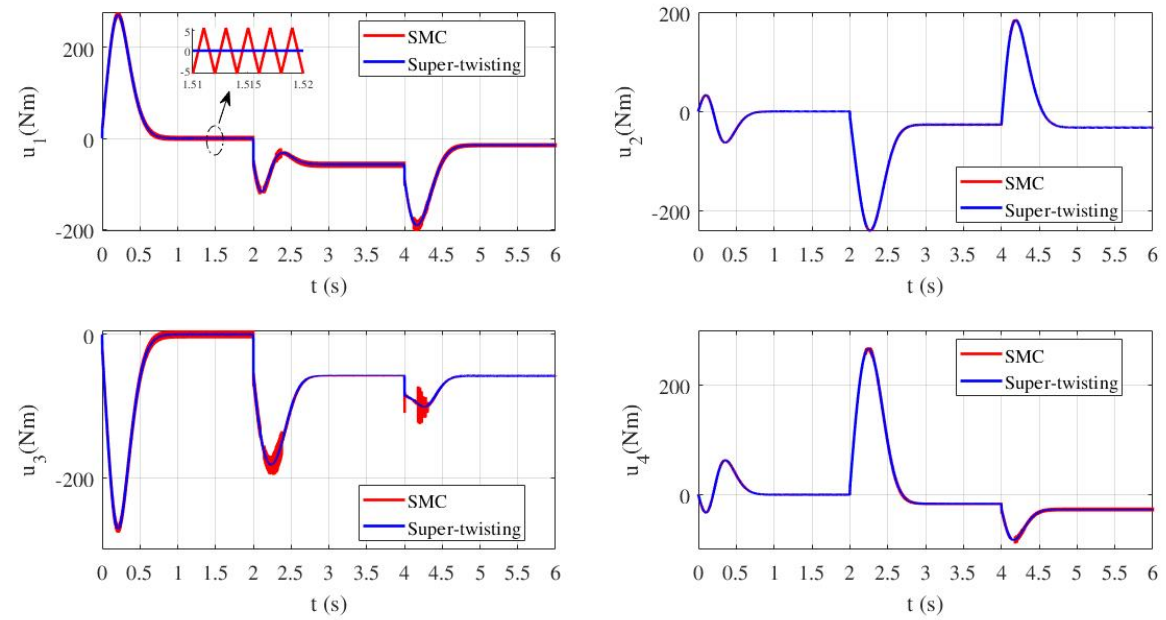

(a) Torque control of the joints
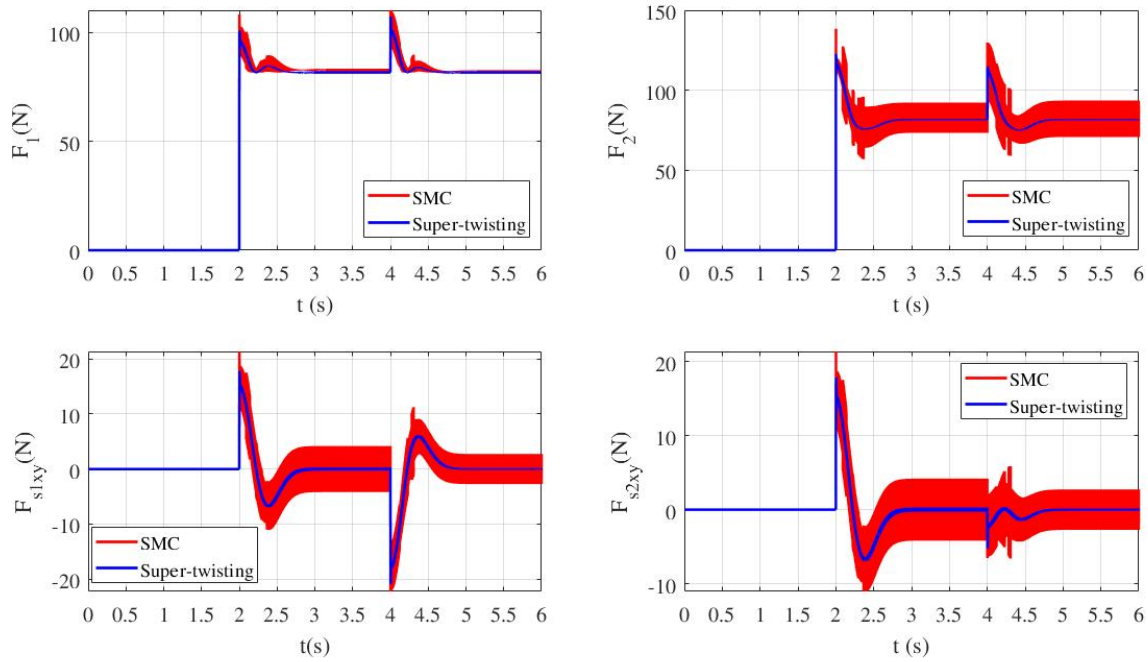

(b) Interaction forces

Figure 4. Control torques and interaction forces, (a) Torque control of the joints,

(b) Interaction forces 

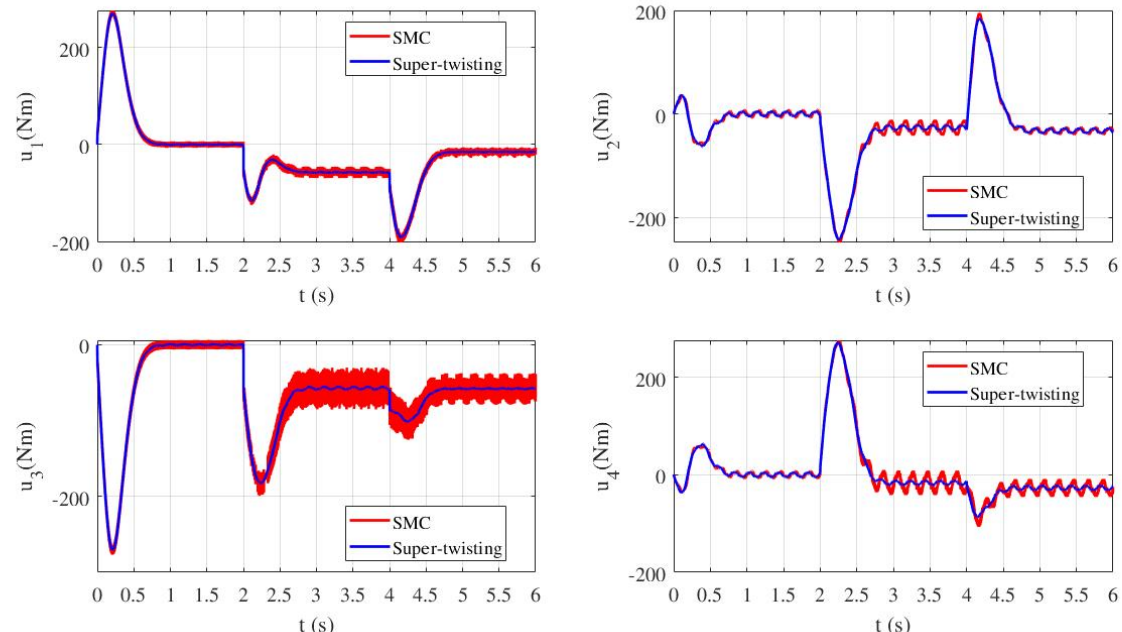

(a) Error tracking of the joint variables
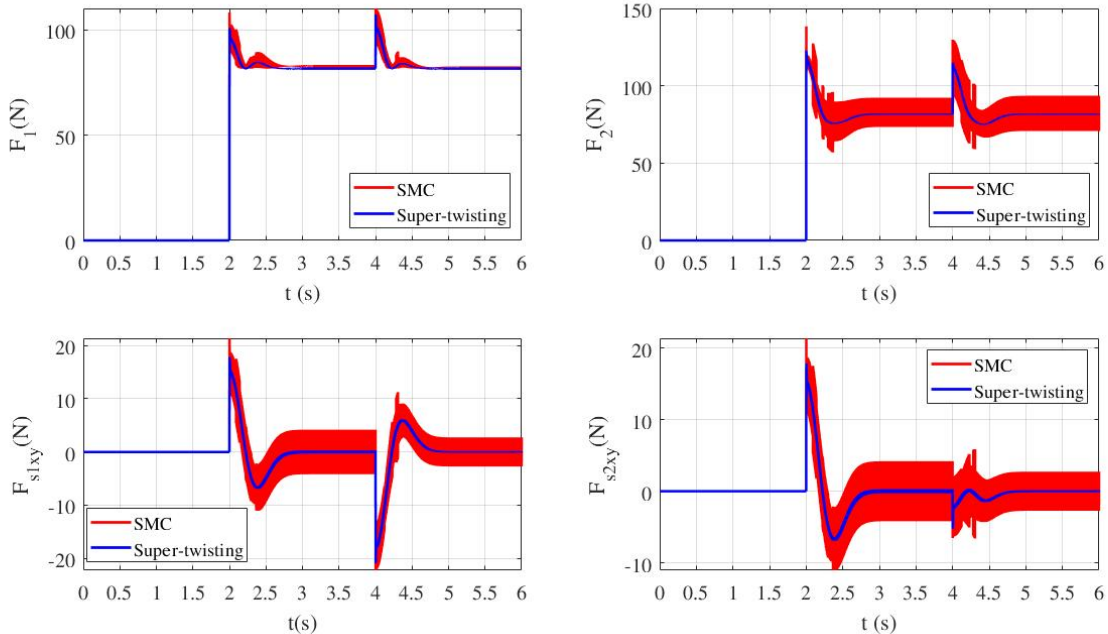

(b) Interaction forces

Figure 5. Result simulation with uncertain disturbance torque, (a) Error tracking of the joint variables,

(b) Interaction forces

Table 1. Simulation parameters of model

\begin{tabular}{cccc}
\hline$m_{1}=m_{3}=5(\mathrm{~kg})$ & $L_{1}=L_{3}=0.5(\mathrm{~m})$ & $d_{2}=0.4(\mathrm{~m})$ & $b_{1}=b_{2}=b_{3}=b_{4}=100(\mathrm{Nms})$ \\
$m_{2}=m_{4}=4(\mathrm{~kg})$ & $L_{2}=L_{4}=0.4(\mathrm{~m})$ & $d_{1}=0.2(\mathrm{~m})$ & $m_{\text {load }}=0.5(\mathrm{~kg})$ \\
$I_{1}=I_{3}=0.1\left(\mathrm{kgm}^{2}\right)$ & $k_{1}=k_{3}=0.25(\mathrm{~m})$ & $\mu=0.3$ & $g=9.8\left(\mathrm{~m}^{2}\right)$ \\
$I_{2}=I_{4}=0.08\left(\mathrm{kgm}^{2}\right)$ & $K_{2}=K_{4}=0.2(\mathrm{~m})$ & & \\
\hline
\end{tabular}

\section{CONCLUSION}

The paper successfully built a generalized model for model $2 n$ degrees of freedom. According to an analysis of the numerical results, the super-twisting sliding mode controller shows adaptability external disturbances, trajectory tracking while the robot handling and transportation the object. Furthermore, the super-twisting sliding mode controller has restricted significantly chattering phenomenon which is the difficult problem of the basic sliding mode controller.

\section{ACKNOWLEDGEMENT}

This research is supported by Hanoi University of Science and Technology research grant T2018-PC-053. 


\section{REFERENCES}

[1] Z. Li, et al., "Brain-actuated Control of Dual-arm Robot Manipulation with Relative Motion," IEEE Transactions on Cognitive and Developmental Systems, vol. 11, no. 1, pp. 51-62, 2017.

[2] R. Ozawa, et al., "Control of an object with parallel surfaces by a pair of finger robots without object sensing," IEEE Transactions on Robotics, vol. 21, no. 5, pp. 965-976, 2005.

[3] J. K. Lee, et al., "Development of a Control System for Master-Slave Operation," ICCAS, vol. 2, pp. 1851-1854, 2010.

[4] C. Natale, et al., "Robust hybrid force/position control with experiments on an industrial robot," IEEE/ASME International Conference on Advanced Intelligent Mechatronics (Cat. No. 99TH8399), no. 5, pp. 956-960, 1999.

[5] J. D. La Fuente, et al., "Handbook on Robotics," Siciliano, editor, vol. 25, no. 9, pp. 1682-1690, 2014.

[6] W. S. Pambudi, et al., "Simulation design of trajectory planning robot manipulator," Bulletin of Electrical Engineering and Informatics, vol. 8, no. 1, pp. 196-205, 2019.

[7] R. T. Yunardi, et al., "Robotic leg design to analysis the human leg swing from motion capture," Bulletin of Electrical Engineering and Informatics, vol. 6, no. 3, pp. 256-264, 2017.

[8] C. Smith, et al., "Dual arm manipulation - A survey," Robotics and Autonomous systems, vol. 60, no. 10, pp. 1340-1353, 2012.

[9] X. Zhou, et al., "Closed-Chain Manipulation of Large Objects by Multi-Arm Robotic Systems," IEEE Robotics and Automation Letters, vol. 2, no. 4, pp. 1832-1839, 2017.

[10] Yuri Shtessel, et al., "Text book: Sliding Mode Control and Observation," New York: Springer New York, pp. 1-42, 2014.

[11] X. Chu, et al., "Flexible robot platform for sample preparation automation with a user-friendly interface," IEEE International Conference on Robotics and Biomimetics, pp. 2033-2038, 2016.

[12] H. Fleischer, et al., "Integration of Electronic Pipettesinto a Dual-arm Robotic System for Automated Analytical Measurement Processes," IEEE 14th International Conference on Automation Science and Engineering, pp. 22-27, 2018.

[13] X. Chu, et al., "Efficient application of dual-arm robots in analytical measurements using motion frames," IEEE International Instrumentation and Measurement Technology Conference, pp. 1-8, 2018.

[14] T. Panagiota, et al., "Robotized assembly process using dual arm robot," Procedia CIRP, vol. 23, no. 3, pp. 47-52, 2014.

[15] D.I. Park, et al., "Development of dual arm robot platform for automatic assembly," 14th International Conference on Control, Automation and Systems, pp. 319-321, 2014.

[16] Hyun Min Do, et al., "Automation of cell production system for cellular phones using dual-arm robots," The International Journal of Advanced Manufacturing Technology, vol. 83, no. 5, pp. 1349-1360, 2016.

[17] X. Yun and V. Kumar, "An approach to simultaneous control of trajectory and interaction forces in dualarm congurations," IEEE Transactions on Robotics and Automation, vol. 7, no. 5, pp. 618-625, 1991.

[18] N. Sarkar, et al., "Dynamic control of 3-drolling contacts in two-arm manipulation," IEEE Transac-tions on Robotics and Automation, vol. 13, no. 3, pp. 364-376, 1997.

[19] S. T. Lin and A. K. Huang, "Position-based fuzzy force control for dual industrial robots," Journal of Intelligent and Robotic Systems, vol. 9, no. 4, pp. 393-409, 1997.

[20] Z. Liu, et al., "Adaptive neural control for dual-arm coordination of humanoid robot with unknown non linearities in output mechanism," IEEE Transactions on Cybernetics, vol. 45, no. 3, pp. 507-508, 2014.

[21] N. Yagiz, et al., "Load transportation by dual arm robot using sliding mode control," Journal of Mechanical science and Technology, vol. 24, no. 5, pp. 1177-1184, 2010.

[22] L. A. Tuan, et al., "Parameter estimator integrated-sliding mode control of dual arm robots," International Journal of Control, Automation and Systems, vol. 15, no. 6, pp. 2754-2763, 2017.

[23] Mark W. Spong, et al., "Robot Dynamic and control second edition," John Wiley and Sons, Inc., 2004.

[24] A . Levant, "Sliding order and sliding accuracy in sliding mode control," International Journal of Control, vol. 58 , no. 6, pp. 1247-1263, 1993.

[25] Awatif Guendouzi, et al., "Higher Order Sliding Mode Control of Robot Manipulator," The Ninth International Conference on Autonomic and Autonomous Systems, 2013. 


\section{BIOGRAPHIES OF AUTHORS}

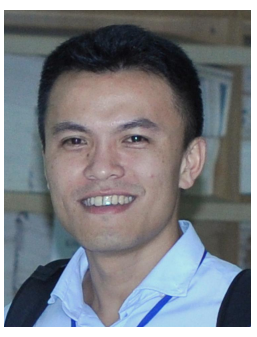

Tung Lam Nguyen received the B.S degree in Control and Automation Engineering from Hanoi University of Science and Technology, Hanoi, Vietnam, 2005, the M.S degree from Asian Institute of Technology, 2007, and the Ph.D from The University of Western Australia, 2014. He is current working as a lecturer at Department of Industrial Automation, School of Electrical Engineering, Hanoi University of Science and Technology. His research interests include motion control, control system and its applications.

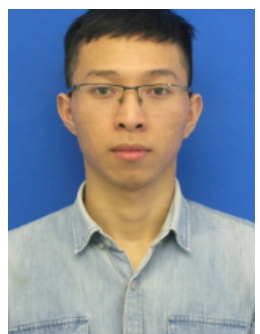

Huu Tinh Vu received the B.S degree in Control and Automation Engineering from Thuyloi University, Hanoi, Vietnam, 2018. He is current working as a student for M.S degree at Department of Industrial Automation, School of Electrical Engineering, Hanoi University of Science and Technology. His researches are in fields of electronics, digital systems, signal processing, manipulator systems. 